\title{
Getting drugs through small pores: Exploiting the porins pathway in Pseudomonas aeruginosa.
}

Susruta Samanta ${ }^{1,5, \dagger}$, Igor Bodrenko ${ }^{1, \dagger}$, Silvia Acosta-Gutiérrez ${ }^{1}$, Tommaso D’Agostino ${ }^{1}$, Monisha Pathania ${ }^{2}$, Ishan Ghai ${ }^{3}$, Christian Schleberger ${ }^{4}$, Dirk Bumann ${ }^{4}$, Richard Wagner ${ }^{3}$, Mathias Winterhalter ${ }^{3}$, Bert van den Berg ${ }^{2}$, Matteo Ceccarelli ${ }^{1^{*}}$

\section{Affiliations:}

${ }^{1}$ Department of Physics, University of Cagliari, SP Monserrato-Sestu Km 0.8, Monserrato, 09042, Italy.

${ }^{2}$ Institute for Cell and Molecular Biosciences, The Medical School, Newcastle University, Newcastle upon Tyne, NE2 4HH, United Kingdom.

${ }^{3}$ Department of Life Sciences and Chemistry, Jacobs University Bremen, 28719 Bremen, Germany.

${ }^{4}$ Biozentrum, University of Basel, Klingelbergstrasse 50/70, CH-4056 Basel, Switzerland

${ }^{5}$ Department of Chemistry, Manipal University Jaipur, VPO Dehmi Kalan, Jaipur, PIN303007, Rajasthan, India.

Corresponding author’s email address: matteo.ceccarelli@dsf.unica.it

${ }^{\dagger}$ These authors contributed equally to the work

Understanding molecular properties of outer membrane channels of Gram-negative bacteria is of fundamental significance as they are the entry point of polar antibiotics into bacteria. Outer membrane proteomics revealed OccK8 (OprE) to be among the five most expressed substrate specific channels of the clinically important Pseudomonas aeruginosa. The highresolution X-ray structure and electrophysiology highlighted a very narrow pore. However, experimental in vitro methods showed the transport of natural amino acids and antibiotics, among them ceftazidime. We used molecular dynamics simulations to reveal the importance of the physico-chemical properties of ceftazidime in modulating the translocation through OccK8, proposing a structure-function relationship. As in general porins, the internal electric field favors the translocation of polar molecules by gainful energy compensation in the central constriction region. Importantly, the comparatively narrow OccK8 pore can undergo a substrate-induced expansion to accommodate relatively large-sized substrates. 
Keywords: outer membrane, porins, antibiotics transport, crystal structure, molecular simulations

Bacterial antibiotic resistance is an important threat to public health. There is an urgent need for novel drugs to enable successful treatment of infections caused by bacterial pathogens. However, the failure in the discovery of new scaffolds ${ }^{1,2}$ together with the increase of resistance levels pose a tremendous challenge for both scientists and pharmaceutical companies. ${ }^{3,4}$ For Gram-negative bacteria, the permeation of small molecules through the outer membrane $(\mathrm{OM})$ is a major problem still poorly understood. In the last few years, several molecules screened for their in vitro target inhibition ability failed to enter clinical phases because apparently they did not permeate through the $\mathrm{OM}$ in vivo. ${ }^{1}$ This failure is central to an elementary knowledge gap - so far, the molecular steps governing permeation of these small molecules through the OM are unknown. In order to contribute to filling this gap, we have explored in large detail one of the best-established entry points for polar antibiotics in Gram-negative bacteria - the porin pathway. We investigated the clinically relevant case of Pseudomonas aeruginosa $(\mathrm{Pa})$, which lacks non-specific trimeric porins (e.g., OmpC and OmpF in Escherichia coli), and has instead many monomeric substrate-specific porins. ${ }^{5,6}$ The $\mathrm{X}$-ray crystal structures of these channels, conversely to OmpF/OmpC, display, on average, small internal pore radii and electrophysiology experiments pointed out spontaneous fast gating. ${ }^{7}$ As recently reported, the same holds true for the channels in Acinetobacter baumannii $(\mathrm{Ab}){ }^{8}$

In our recent work, we investigated the relationship between the dipole moment of the substrates and their ability to follow the sequence of steps imposed by the internal electrostatics of non-specific porins in E. coli. ${ }^{9-12}$ However, key functional and structural differences between these porins and specific channels require a more detailed investigation to disclose the mechanisms of permeation through the $\mathrm{OM}$ and to define new strategies to design effective antibiotics. The presence of multiple small pore-size channels might offer alternative pathways for antibiotics penetration in $P a$, as recently demonstrated for carbapenem molecules. ${ }^{13}$ Further, small structural modifications of antibiotics displayed dramatic effects on the ability to permeate through the main porin of $\mathrm{Pa}$, OccD1 (OprD). ${ }^{14}$

Recently, the FDA has approved a combination of ceftazidime and the $\beta$-lactam inhibitor avibactam for treatment of $\mathrm{Pa}$ infections. ${ }^{15,16}$ In this context the important question remains how do comparatively large drug molecules, like ceftazidime, enter the bacterial cell via small-pore channels? In this work, we are focusing on the analysis of OccK8 (also known as 
OprE), a member of the outer membrane carboxylate channel (Occ) family of $P a^{5,6}$ Previously, OccK8 was already proposed as a possible entry pathway for the translocation of $\beta$-lactams antibiotics, ${ }^{17}$ especially cephalosporins like ceftazidime that carry two negative charges. ${ }^{18,19}$

In the present study, we combined information from proteomics, liposome swelling assays (LSA), electrophysiology, X-ray crystallography, and molecular dynamics simulations to understand the molecular steps of the transport of substrates and antibiotics within the framework of the porin structure. We compared OccK8 with the well-studied large-pore channel OmpF from E.coli and use the different physico-chemical properties of the antibiotics to explore the chemical space within the pore. This allowed us to explore the mechanism of transport and determine the key structural and dynamical elements controlling permeation through this family of channels.

\section{Results and discussion.}

Expression levels of OccK8 in $\mathbf{P a}$. To quantify the abundance of OccK8 in $\mathrm{Pa}$ UCBPPPA14 in infected lung tissues, we employed a sensitive targeted proteomics approach (parallel reaction monitoring) on a high resolution and accurate mass instrument with absolute quantification using heavy-isotope labeled reference peptides (see SI for extended information). The results showed that in both mouse and rat pneumonia models, OccK8 was present at 300-2000 molecules per $\mathrm{Pa}$ cell, making OccK8 one of the five most abundant simple porins. As a comparison, the most abundant porin, OprD, has 10,000 - 27,000 molecules per cell.

Permeation assay through OccK8. In order to characterize the OccK8 transport mechanism, we investigated the permeation of different molecules using two independent techniques: liposome swelling assays (LSA) and electrophysiological reversal potential measurements. $^{20,21}$ The measured rate of swelling of liposomes with reconstituted porins is correlated with the rate of influx of molecules through porins. We tested the permeability of OccK8 and OmpF against selected natural amino acid residues with different electrostatics: positively charged arginine, negatively charged glutamic acid, and neutral glycine. We also assessed three cephalosporins and two penicillins scanning different electrostatic and size: carbenicillin (doubly negative and small), cefotaxime (negative), ceftazidime (negative with a positive group), cefepime (zwitterionic), and ampicillin (zwitterionic). The LSA results are summarized in Figure 1, and chemical structures of the compounds are shown in Figure S4 (see also Methods). 
Arginine and glutamate show comparable permeation rates, indicating OccK8 is not very selective for carboxylate-containing positively or negatively charged small molecules (Figure 1). We also observed that certain molecules with charged groups of both polarities in their structure, i.e., cefepime (zwitterionic) and ceftazidime (negatively charged) have relatively high permeation rates through OccK8. Interestingly, compared to the other substrates, these two molecules permeate similarly through OccK8 and OmpF, even though the latter has a larger pore size. In contrast, ampicillin, though also zwitterionic, shows poor permeation through OccK8, and comparable to cefepime in OmpF. Finally, negatively charged cefotaxime, and di-anionic carbenicillin, reveal very poor permeation through OccK8 (Figure 1). The "negative" permeation values for these two compounds result from channel-mediated outward-directed water flux (i.e. liposome shrinking). This effect is very small, and only evident in the absence of significant uptake of substrate that causes liposome swelling. The substrate uptake rates for such cases are thus effectively zero.

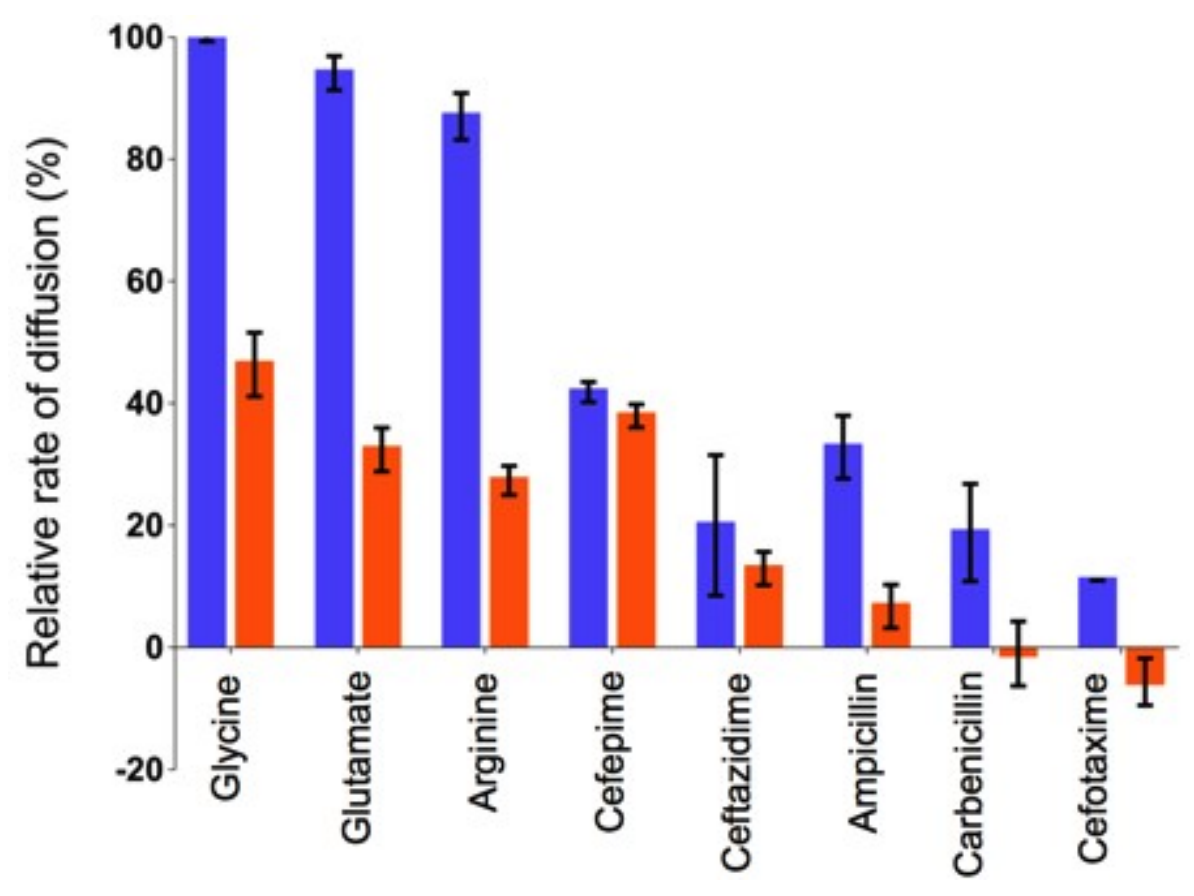

Figure 1. In vitro transport by OccK8 and OmpF. Liposome swelling data for various small molecule substrates. Transport of glycine through OmpF is set to $100 \%$ for standardisation. Substrate concentrations ranged from 4-12 mM (see Methods). The substrate uptake rates were averaged from the duplicate values measured from three different liposome preparations made on different days. Values correspond to averages and their standard deviations $(n=6-9)$. The difference in uptake rates for ceftazidime between OmpF and OccK8 are statistically not significant $(\mathrm{P}=0.09)$. 
Electrophysiological permeation assay of antibiotics through OprE. To gain further information on permeation through OccK8, we reconstituted it into planar lipid bilayers. We first characterized the pore conductance in $100 \mathrm{mM} \mathrm{NaCl}$ solution. OccK8 shows very low conductance values $\left(G_{\text {OccK } 8}^{\text {monom }}=13 p S i\right.$, significantly smaller than the general porin OmpF ( $\dot{G}_{\text {OmpF }}^{\text {monomer }}=178 \mathrm{pS}$ ), but also lower than classical sodium or potassium channels (e.g. $\dot{G}_{K c s A}=72 p S$ ) at similar salt concetration. ${ }^{22-24}$ However, the conductance ratio of OccK8 with respect to $\operatorname{OmpF}\left(\frac{G_{\text {OccK } 8}^{\text {monomer }}}{G_{\text {OmpF }}^{\text {monomer }}}\right)$ of $0.071(100 \mathrm{mM} \mathrm{NaCl})$ increases at higher ionic strength to $0.167(1 \mathrm{M} \mathrm{NaCl})$. In a next series of measurement, we determined the OccK8 ion selectivity by imposing a concentration gradient of $600 / 100 \mathrm{mM} \mathrm{NaCl}$ between the two compartments. Remarkably, in contrast to the other members of the Occ subfamily (OccK1-7) that show a conserved selectivity for anions, ${ }^{6}$ OccK8 revealed a slight preference for transporting cations $\left(P_{N a^{+i} / P_{C r^{i}=1.5 i} i}\right.$, Table 1, Table S1).

In further series of measurements, we investigated the relative permeability of charged molecules through OccK8. For this we reconstituted the channel into a symmetric $10 \mathrm{mM}$ $\mathrm{NaCl}$ solution, in a second step we increased the Na-Substrate (or Substrate-Cl) concentration on one side up to $50 \mathrm{mM}$ (Table 1), creating the condition for both $\mathrm{Na}(\mathrm{Cl})$ and substrate transport through OccK8, or a current of charges. This concentration gradient resulted in a Nernst potential $V_{\text {Nerst }}=56 \mathrm{mV}$ for sodium $\left(\mathrm{V}_{\text {Nerst }}=-56 \mathrm{mV}\right.$ for chloride). By applying an additional external electric potential, we can determine the potential at which the total current is zero, called the reversal potential. A value of $(V i \mathrm{i} r e v) i$ close to the $V_{\text {Nerst }}$ means low transport rate for substrates, close to 0 high transport rate. From Table 1 we can conclude that all anionic compounds permeate through OccK8, albeit to varying degrees, confirming the general trend of LSA data. By fitting these $V_{\text {rev }}$ values using the Goldman-Hodgkin-Katz (GHK) voltage equation ${ }^{23,25,26}$ (details in the SI), we obtained the relative permeability value for the different substrates, their conductance and the transport rates (Table 1). Considering the small size of the OccK8 channel and the large sizes of the different substrates, the conductance values (at $V_{m}=10 \mathrm{mV}$ in Table 1) show that OccK8 reveals a surprisingly good permeability for the antibiotics. By extrapolating to a more physiological gradient concentration of $10 \mu \mathrm{M}$, we estimated the different translocation rates (molecules/s) through a single OccK8 channel (see Table 1 and SI for details). 
OccK8 Architecture. Porins of the OccK family (in particular OccK1 to OccK7) have relatively small hourglass-shaped pores with positively charged residues in the central constriction region, and they are anion selective. ${ }^{6}$ Regarding the eighth member of the family - OccK8, there is a lack of structural information. OccK8 shows a high level of sequence variation with the other members of the same family, mainly in the external loops. The external loops are in contact with the external medium and they account for the preference of OccK8 towards specific chemical environments, as reported in the literature. ${ }^{27}$ We therefore solved the structure of OccK8 using data to a resolution of $1.9 \AA$ by X-ray crystallography (PDB code: 4FRX; Table 2). The OccK8 structure is shown in Figure 2. The constriction region created by internalized extracellular loops L3 and L7 (orange and yellow in Figure 2b) has a much smaller cross-section area ( 9 $\AA^{2}$ ) compared to that of OmpF (25 $\AA^{2}$, Figure 3A). As already reported for other porins, ${ }^{10,} 28$ the constriction region is characterized by the socalled charge segregation: a basic ladder consisting of arginine (R163, R131, R421) and lysine (K397) residues on one side, and two negatively charged aspartate residues on the opposite side, located neat Gate A (D311) and near Gate B (D322) respectively (Figure 2c). In this case, the two negative residues (D311, D322) are part of the internal loop L7. L7 flexibility is very different from loop L3 in general porins and it might affect the passage of substrates, ${ }^{10,20}$ as recently shown for Occ channels of A. baumannii. ${ }^{29}$ Moreover, the negatively charged glutamate residue (E419), and the polar glutamine residue (Q324) play an important role in forming and modulating the constriction region in this channel (Figure 2b). It is worth noting that D322, the negative residue at the exit of the constriction region, is conserved in only 3 members (OccK1, OccK2, and OccK9) out of 11 known Occ channels (OccK1-11) (Figure S1).
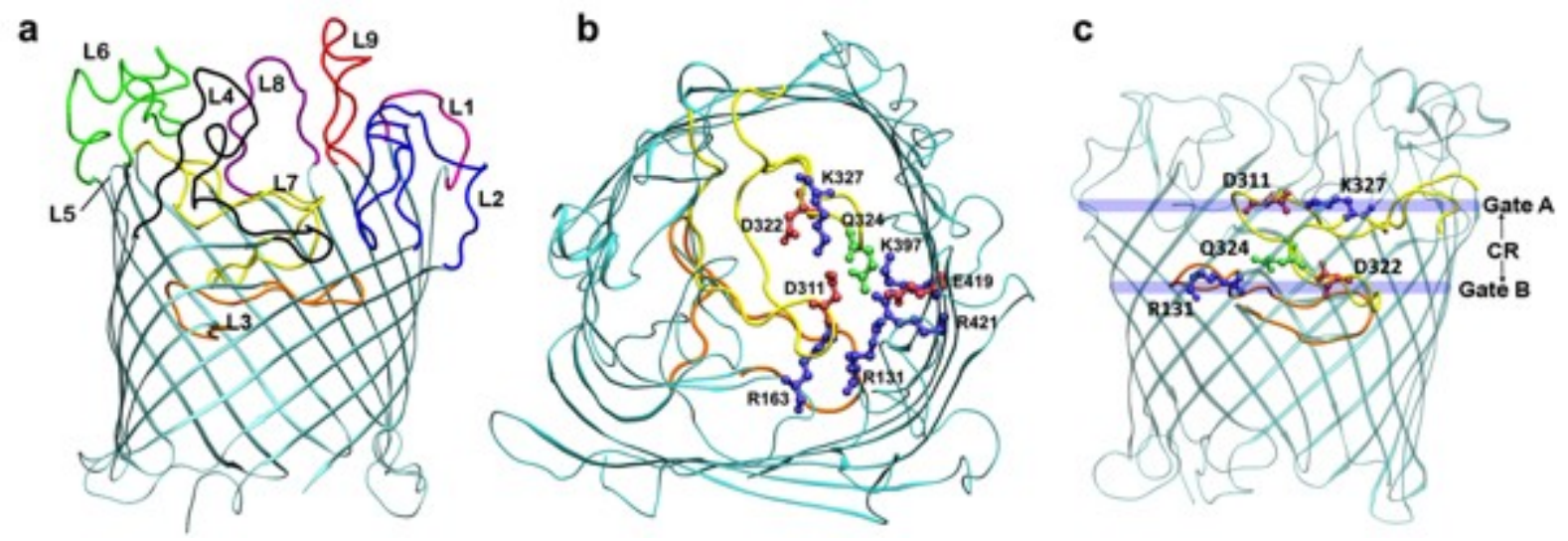
Figure 2. (a) Structure of OccK8 with the important loops indicated. (b) Definition of the key residues in the constriction region. (c) Location of the two Gates A and B. Salt bridge between D311 and K327 is highlighted in orange. The loops L3 and L7 are shown in orange and yellow, respectively.

Based on the pore size profile calculated along the z-axis of diffusion, we can identify two distinct gates: Gate A and Gate B, towards the extracellular and the periplasmic side of the channel respectively (Figure 2c). These two gates border the constriction region (Figure 2c, Figure 3a) along the axis of diffusion through the pore. While Gate B is located halfway down the diffusion axis as in other porins, ${ }^{30}$ Gate A controls the entry to the constriction region. Gate A is modulated by a salt bridge between a lysine (K327) of the basic ladder and a glutamate (D311) residue of loop L7 (Figure 2 b and c, highlighted in orange). The sequence alignment (Figure S1 in the supplementary information) of OccK8 with other members of the Occ family shows that other Occ porins have neither a basic residue corresponding to K327, nor an acidic residue corresponding to D311, ${ }^{6}$ the latter being part of an insertion in the sequence (see Figure S1A). This unique salt bridge is unstable and easily disrupted in the presence of $\mathrm{KCl}$, as shown by simulating OccK8 at different salt concentration (Figure S2). The increase of the pore size correleates with a higher current measured by electrophysiology experiments at higher ion concentration. Thus, these two residues might play a significant role in binding and directing the substrates towards the constriction region, analogous to the two arginine residues (R167 and R168) located in the preorientation region of $\mathrm{OmpF} .^{31}$

Steric factor inside the channel. In order to identify the role of both gates on molecular transport, we performed metadynamics simulations to study the translocation of a neutral (hydrophobic) sphere of radius $3.5 \AA$ (size similar to that of avibactam ${ }^{20}$ ) through OccK8 and, for comparison, through OmpF.

Both porins have a pore smaller than the spherical probe at equilibrium. However, by quantifying the free energy barrier for translocation of the probe, we observed a lower value through the smaller OccK8 $(6.7 \mathrm{kcal} / \mathrm{mol})$ than through the larger OmpF channel $(10.8 \mathrm{kcal} /$ mol) (Figure 3b). This indicates that the constriction region in OccK8, though smaller in the crystal structure, is rather dynamic and flexible (loop L7), while it is more rigid in OmpF (loop L3). The barrier for translocation of the probe corresponds to the minimum of the pore size in both OmpF and OccK8, located respectively at Z=-2 $\AA$ and around Gate B at Z=+2 $\AA$ (Figure 3a). Apparently both porins have a filter controlling transport mainly by size. 

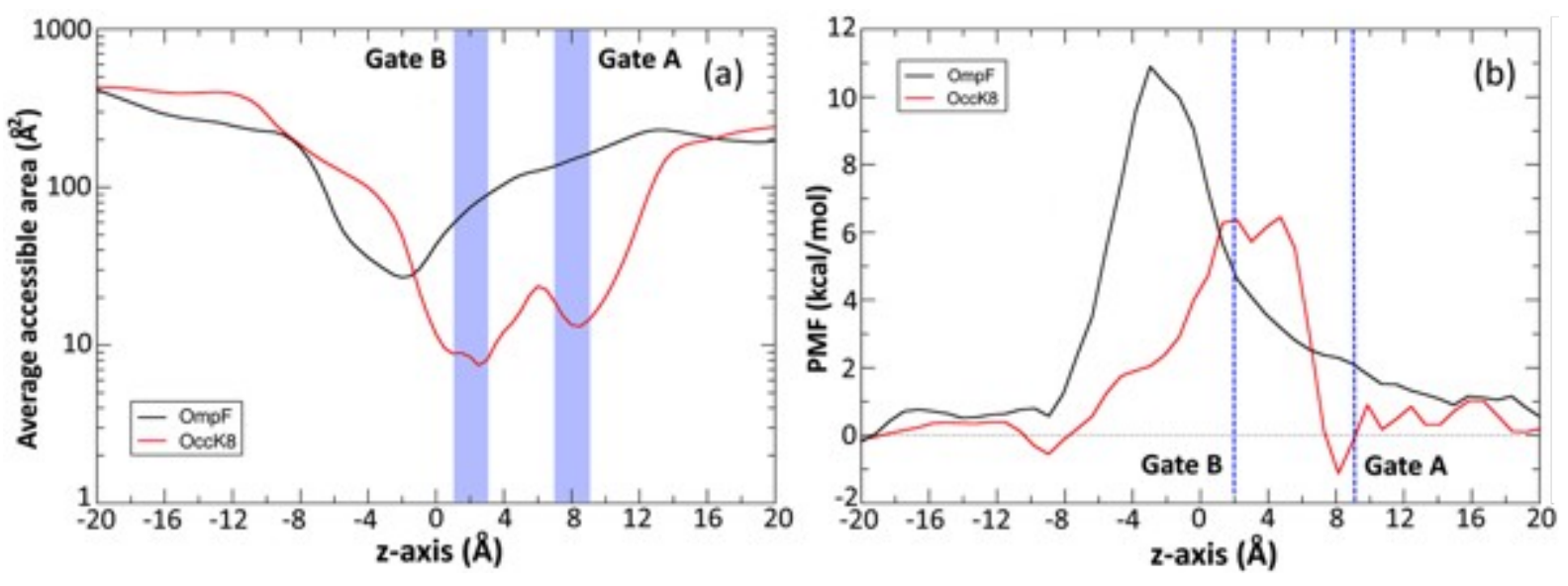

Figure 3. (a) Comparison of the average accessible area of the OmpF and OccK8 pores along the channel z-axis calculated with standard molecular dynamics simulations. (b) Energy barriers for the percolation of a neutral sphere (radius $=3.5 \AA$, cross section $=38.5 \AA^{2}$ ) through the porins calculated with metadynamics simulations. The two gates in OccK8 are depicted as blue shades.

Simulations of substrate translocation. In order to determine the role of the internal electrostatics of the pore in transport of molecules, we studied the translocation of the three selected amino acids (glycine, glutamate, and arginine) through OccK8 using metadynamics simulations. ${ }^{32}$ The reconstructed free energy surfaces (FESs) for the translocation of the substrates are shown in Figure 4 with respect to the two reaction coordinates (also known as collective variables) used in metadynamics: the orientation of substrates ( $\mathrm{x}$ axis) and their positions along the axis of diffusion of the pore ( $\mathrm{y}$ axis). The free energy surfaces provide us with two important features - the energy barrier of permeation, and the affinity of the molecules with respect to the two reaction coordinates. Arginine and glutamic acid show similar barriers for translocation through OccK8 (6.7 kcal/mol and $6.8 \mathrm{kcal} / \mathrm{mol}$ respectively), while the barrier for the small glycine is smaller ( $3.3 \mathrm{kcal} / \mathrm{mol})$. These energy values correlate well with the translocation rates indicated by the liposome swelling assay data (Figure 1), which also show similar permeation rates for arginine and glutamic acid. 

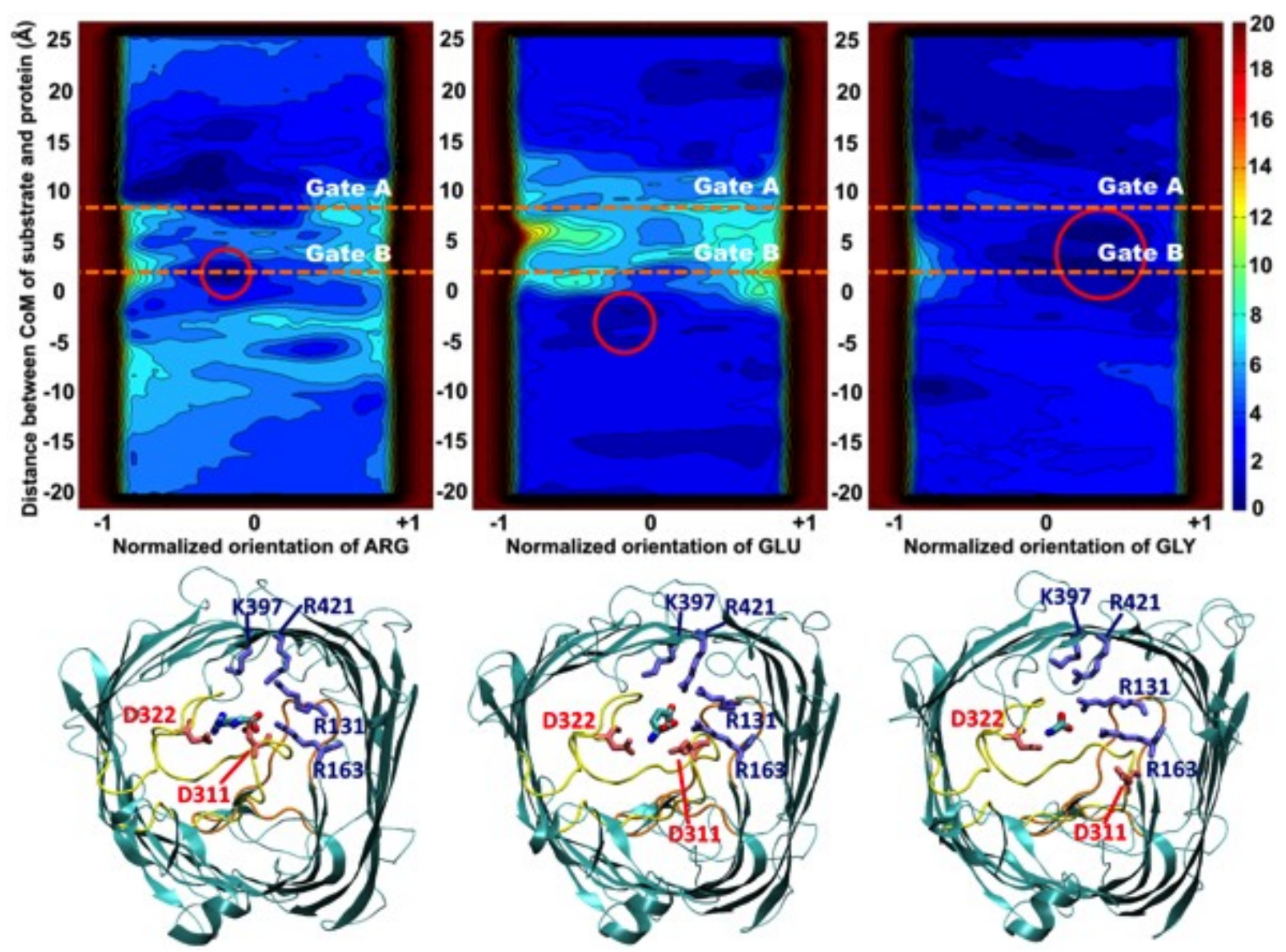

Figure 4. FESs of the translocation of (top, left to right) arginine, glutamic acid, and glycine through OccK8. Each color represents an energy difference of $1 \mathrm{Kcal} / \mathrm{mol}$. The minima in the FESs are shown in red circles. The structures corresponding to the minima in the FESs of the translocation are shown in the lower panels.

For both the neutral (glycine) and the positively charged (arginine) amino acids the constriction region (Figure 4, upper panel) is energetically more favourable than for the negatively charged (glutamic acid) one, in agreement with the slight cation selectivity of the pore. Indeed, glycine exhibits a wide minimum in the constriction region between the two gates, probably caused by its small size. The porin-substrate conformation corresponding to the highlighted minima on the free energy map (Figure 4, bottom panels) for each substrate show that the acidic and basic residues of the constriction region play a crucial role in the favourable interactions defining the minima. Residue D322 creates a recognition pocket by interacting with the positive charged group of all selected substrates. This residue is conserved only in three other channels among the eleven of the Occ subfamily: OccK1-2, and OccK9 (Figure S1). For glycine and glutamic acid the negatively charged part is closed to the basic ladder (R163, R131, R421, and K397) (Figure 4, bottom panels). Thanks to the 


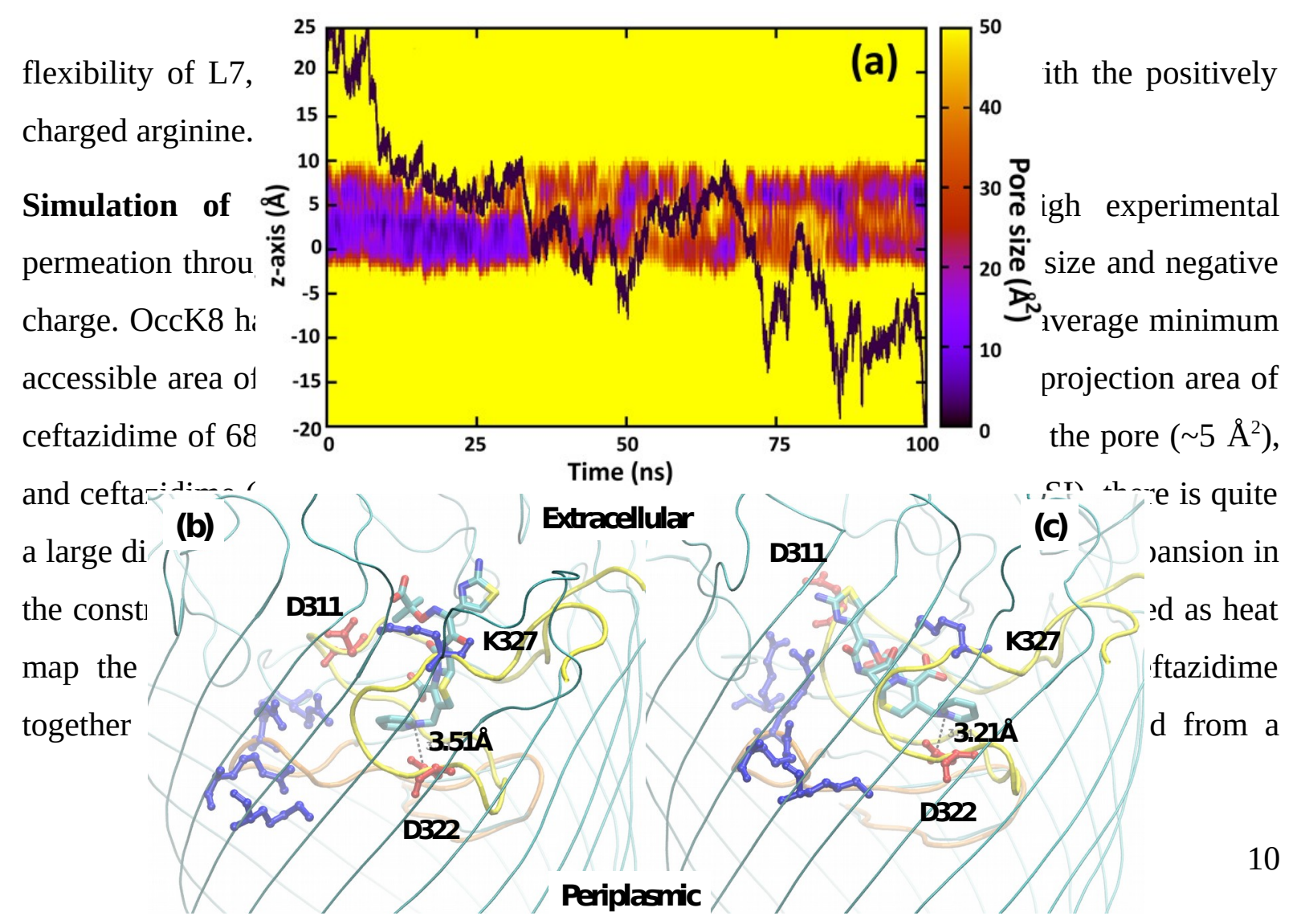

Figure 5. (a) Temporal evolution of the cross section of OccK8 during the translocation of 
metadynamics run. The constriction region remains narrow until ceftazidime approaches. We observe an alternate widening and narrowing of gates $\mathrm{A}$ and $\mathrm{B}$ that helps to accommodate ceftazidime in the constriction region, facilitating translocation. First, Gate A widens, allowing ceftazidime to enter the constriction region. Then, when ceftazidime approaches the constriction region and its positive group binds the recognition pocket (created by D322, see Figure $5 b$ and $5 c$ ), the Gate B widens while the Gate A narrows. Therefore, the recognition motif (D322) helps to widen Gate B upon binding of the positive group of the molecule.
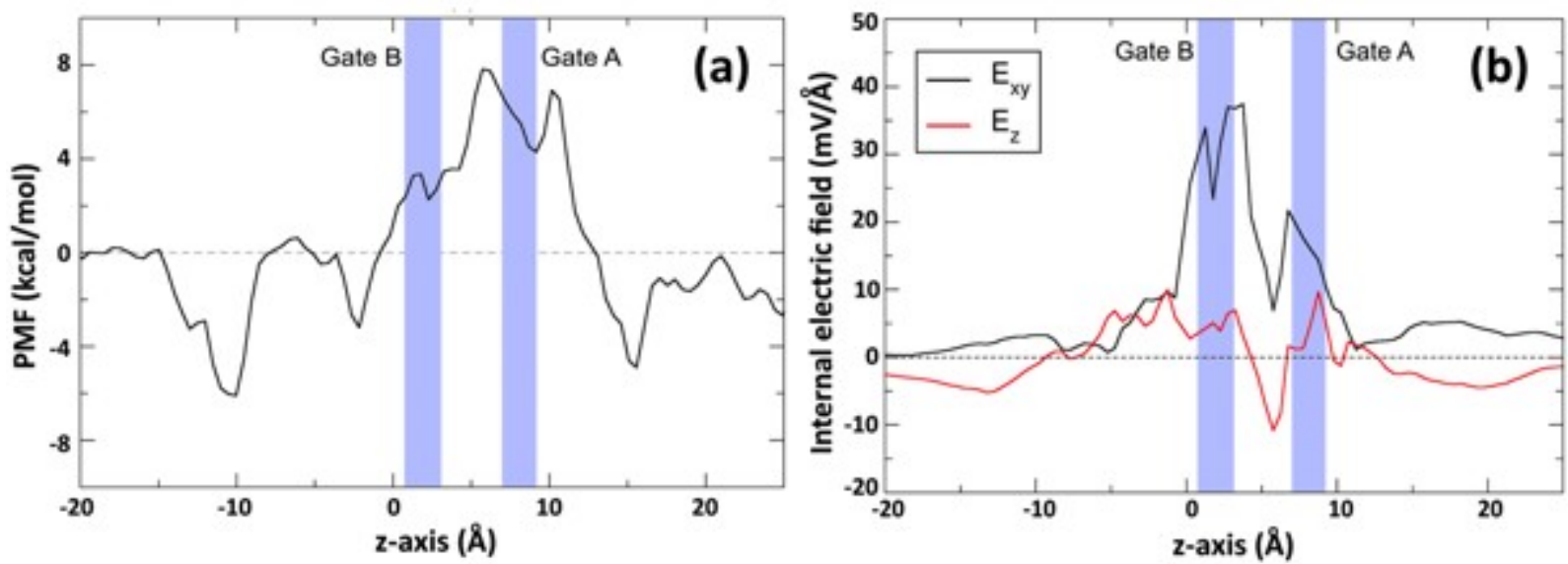

Figure 6: (b) Potential of mean force (PMF) profile for the translocation of ceftazidime through OccK8. For clarity we reported the locations of the gates depicted as blue shades. (c) The macroscopic internal electric field of OccK8. The transversal component is shown in black, and the longitudinal component parallel to the axis of diffusion is shown in red. The constriction region lies in between the two gates shown in blue shades.

Because of the abovementioned segregation of charges across the pore constriction, we expect a strong internal electric field, which was evaluated from standard MD simulations. ${ }^{11}$ Similarly to other non-specific porins previously investigated, the highest intensity of the macroscopic electric field is located in the constriction region in the transverse direction (XY) with respect to the diffusion axis (Z). ${ }^{11,28}$ The strength is comparable to that of OmpF ( 36 $\mathrm{mV} / \AA^{11}$ ), while the longitudinal component $(\mathrm{Z})$ is negligible. Since the transversal component of the electric field has a peak near the two gates (Figure 6c), these regions can also be classified as electrostatic gates. The absence of a barrier from the $1 \mathrm{D}$ free energy profile near Gate B (Figure 6b) indicates that the expected steric effect is completely compensated by electrostatics. The minimum in the free energy surface of ceftazidime (Figure S3) near Gate B (where the transversal component has a maximum) consists of two different conformers (Figure 7, and Figure S3 state 1 and state 2). In both conformers, the 
positive group is close to D322. In the former (state 1), the lateral $\mathrm{COO}^{-}$(the carboxyl group not attached to the $\beta$-lactam ring) interacts with $\mathrm{K} 327$, while in the latter it interacts with the basic ladder. For both conformers, the permanent dipole moment of the molecule is oriented along the transversal electric field (Figure 7). In large non-specific porins like OmpF/OmpC or the Major Outer Membrane Porin of Campylobacter jeujenii, the transversal electric field imposes a preferred orientation for the dipole moment of translocating molecules in the constriction region, ${ }^{11,28}$ as for ceftazidime here.
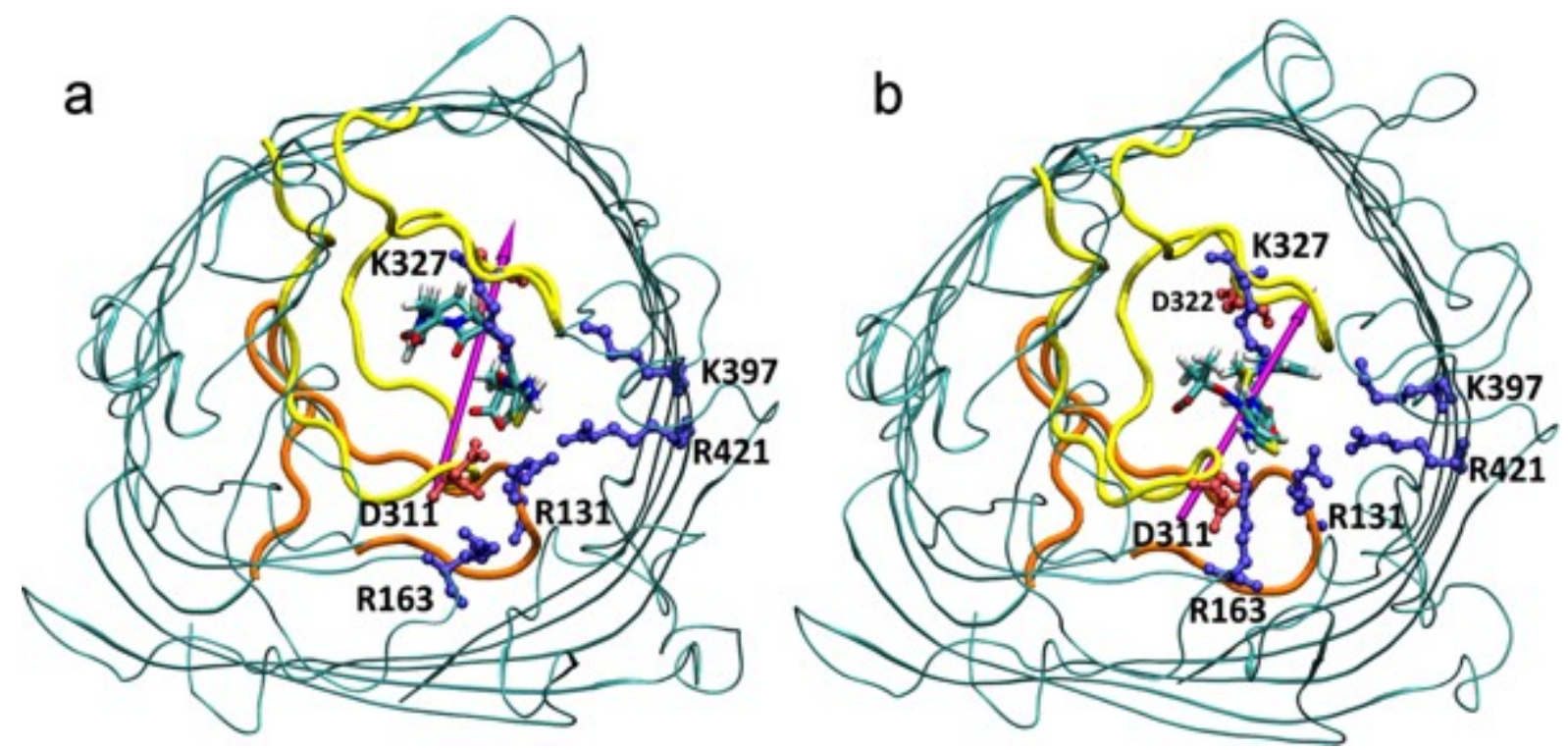

Figure 7 Orientation of the dipole of ceftazidime in the minima for the two relevant conformational clusters: (a) state 1, and (b) state 2. In both cases, though the orientation of the ceftazidime molecule is different, the dipole points in the same direction.

When comparing ceftazidime to the other molecules used in LSA, only cefepime has a high permeation and might bind to the recognition pocket because it is structurally similar to ceftazidime (Figure S4). The presence of both a positive and a negative group allow ceftazidime and cefepime to interact at the same time with the recognition pocket near gate $\mathrm{B}$, represented by the motif D322, and the basic ladder. This is not the case for cefotaxime and carbenicillin, which do not have any positive group. LSA and electrophysiology experiments show, indeed, that these are the molecules with the lowest permeation. Ampicillin shows a better permeation than cefotaxime and carbenicillin, but nevertheless, worse than ceftazidime and cefepime. Although ampicillin has a positive group in the scaffold and thus is zwitterionic like cefepime, it has its permanent dipole moment oriented along its main axis of inertia, with the two charged groups too far apart (8.5 $\AA$ ) to interact with the recognition pocket and the basic ladder simultaneously. In contrast, the positively charged group of 
cefepime and ceftazidime has an average distance from the nearest negatively charged group of $3.8 \AA$. This distance is not much larger than the distance between the positive and negative groups in the three tested amino acids, on average $2.6 \AA$ (Figure S5), which fit well in the recognition pocket near gate $B$. The balance between the need of the molecule to align accordingly to the electric field and to fit in the constriction region was shown already for non-specific channels, and defines the magnitude of the barrier encountered during the translocation..$^{10,31,34}$

\section{Conclusion.}

In this study we investigated the translocation of a set of molecules through OccK8, one of the most highly expressed substrate specific porins in Pseudomonas aeruginosa. Liposome swelling assays and single channel electrophysiology confirmed OccK8 to be an entry point for several antibiotics, including ceftazidime. These observations are surprising, as ceftazidime is a large antibiotic and OccK8 has a very narrow channel compared to the classical trimeric general porins of Enterobacteriaceae.

Starting from the X-ray high resolution crystal structure of OccK8, we analysed with all-atom MD simulations the diffusion of substrates to gain detailed knowledge on the steric and electrostatic effects inside the pore. OccK8 exhibits electrostatic features similar to those of the general porins: a strong transversal component of the electric field in the constriction region, and a significantly weaker longitudinal component. However, our work shows that the constriction region of this specific porin behaves differently from larger general porins. Though small, the constriction region in OccK8 is very flexible displaying two gate-regions, which widen and narrow sequentially to allow the translocation of large molecules.

Combining the diverse results, we demonstrated how these specific porins can serve to translocate large molecules with a suitable chemical structure. The main barrier for translocation arises from the steric effects in the constriction region. This adverse effect can be compensated significantly by favorable electrostatic interactions through the alignment of the dipole moment of the molecule with the intrinsic electric field created by the pore in the constriction region, as already observed in general porins. In addition, a precise complementarity between the recognition pocket and the entering molecule induces the widening of the small but highly flexible constriction region, allowing the passage of suitable large molecules at relatively high rates. To our knowledge, the results demonstrate that OccK8 is the first example of a narrow-channel porin with a highly dynamic constriction 
region that allows transport of substrates that would be too large for the pore observed in the crystal structure.

Our findings open up new possibilities for further research on the substrate specific porins of Gram-negative bacteria and their potential utilization as antibiotic delivery vehicles. Our study gives new molecular descriptors for antibiotic molecules that will allow better permeation through a highly expressed and therefore clinically relevant porin in $P$. aeruginosa. (i) The presence of a negative group in the scaffold helps molecules to slide along the basic ladder, (ii) the presence of a positive group in the scaffold at a specific distance from the negative group helps the molecule to interact with D322 in the recognition pocket, thereby inducing widening of the constriction region. The results of our combined methods show a new, three-step strategy to discover/develop new antibiotics that permeate efficiently: (i) in vivo investigations on metabolite pathways and expression levels to demonstrate relevance of the porin during infection; (ii) crystallization of the channel and in vitro analysis of small-molecule permeation properties; (iii) in silico identification of the rate limiting steps and molecular mechanism of the translocation process.

\section{Methods.}

Materials. Ceftazidime Hydrate and carbenicillin disodium were obtained from Cayman chemicals, cefotaxime sodium, glutamic acid, arginine, $\mathrm{HCl}$, potassium chloride, sodium chloride was obtained from Sigma Aldrich. All other chemicals used were procured from Applichem. 1,2-diphytanoyl-sn-glycero-3-phosphocholine was procured from Avanti Polar Lipids (Alabaster, AL).

Expression and purification of OccK8. The occK8 gene from $P$. aeruginosa was cloned into the E. coli expression vector pB22 with an N-terminal hexa-histidine tag for purification. BL21(DE3) T1 phage-resistant cells (New England Biolabs, Ipswich, MA) were transformed with pB22-occK8. The cells were grown to $\mathrm{OD}_{600} \sim 0.6$ at $37^{\circ} \mathrm{C}$ and then induced with $0.1 \%$ arabinose at $20^{\circ} \mathrm{C}$ overnight. Cells were harvested by centrifugation at $4,500 \mathrm{rpm}$ for $30 \mathrm{~min}$ (Beckman Coulter, J6-MC). Cell pellets were suspended in TSB (20 mM Tris, $300 \mathrm{mM} \mathrm{NaCl}$, 10\% glycerol, $\mathrm{pH} 8.0)$ and cells were lysed by sonication ( $3 \times 40 \mathrm{~s}$ intervals) (Branson Digital Sonifier). Total membranes were obtained by centrifugation at 40,000 rpm for $40 \mathrm{~min}$ (45 $\mathrm{Ti}$ rotor, Beckman L8-70M ultracentrifuge). Membranes were homogenized in TSB and solubilized in 1\% DM (n-Decyl- $\beta$-D-Maltopyranoside, Anatrace, Santa Clara, CA) and 1\% 
LDAO (n-Dodecyl-N,N-Dimethylamine-N-Oxide, Anatrace) for $2 \mathrm{~h}$ at $4^{\circ} \mathrm{C}$ followed by centrifugation at 40,000 rpm for 30 min to remove precipitates and unsolubilized membranes. The membrane extract was applied to a $10 \mathrm{ml}$ nickel column. The column was washed with 10 column volumes (CV) TSB containing $0.2 \% \mathrm{DM}$ and $15 \mathrm{mM}$ imidazole. The protein was eluted with 3 CV TSB containing 0.2\% DM and $200 \mathrm{mM}$ imidazole. OccK8 was further purified by gel filtration chromatography using $10 \mathrm{mM}$ Tris, $50 \mathrm{mM} \mathrm{NaCl}$, and $0.12 \% \mathrm{DM}$, $\mathrm{pH}$ 8.0. For final polishing and detergent exchange, another gel filtration chromatography step was performed in $10 \mathrm{mM}$ Tris, $50 \mathrm{mM} \mathrm{NaCl}$, and $0.35 \% \mathrm{C}_{8} \mathrm{E}_{4}$ at $\mathrm{pH}$ 8.0. The purified protein was concentrated to $\sim 10 \mathrm{mg} / \mathrm{ml}$ using $50 \mathrm{kDa}$ molecular weight cutoff filters (Amicon) and flash-frozen in liquid nitrogen prior to storage at $-80{ }^{\circ} \mathrm{C}$.

Crystallization and structure determination of OccK8. Initial crystallization trials were performed at 293K using a Gryphon crystallization robot (Art Robbins Instruments) and by sitting-drop vapour diffusion using various commercial and in-house screens. The initial hits were optimized by fine-screening with larger drops using various protein-to-mother liquor ratios by hanging drop vapour diffusion. Depending on the conditions, crystals were flashfrozen in liquid nitrogen either directly from the drop or after suitable cryoprotection, typically by adding glycerol to the drop. The OccK8 crystals that gave suitable diffraction data were obtained in 25\% PEG 400, 0.2 M ammonium sulphate, 0.05 M Hepes at pH 7.0. Diffraction data were collected at $100 \mathrm{~K}$ at the National Synchrotron Light Source (Brookhaven National Lab) at beamline X25. Processing was carried out with HKL2000. The OccK8 structure was solved by molecular replacement in Phaser using OccK1 (PDB ID: 2QTK) as the search model. ${ }^{35}$ Model (re)building was performed manually within Coot and the structures were refined using Phenix. ${ }^{32,36}$ Structure validation was performed within Phenix.

Liposome swelling assays. Osmotically active liposomes were prepared as described previously with modifications. A liposome stock solution was made by mixing $100 \mathrm{mg}$ egg phosphatidylcholine (25 mg/ml in chloroform; Avanti Polar Lipids) with $2.3 \mathrm{mg}$ dihexadecylphosphate (Sigma), dissolved in $1 \mathrm{ml}$ of chloroform. For each protein tested, 80 $\mu \mathrm{l}$ from the stock solution was added into a glass vial and dried first under an air stream and afterwards for two hours under a vacuum. Afterwards, the dried film was resuspended in 100 $\mu \mathrm{l}$ water and the protein was added, vortexed, sonicated for $1 \mathrm{~min}$ in a water bath, and dried overnight under vacuum. The amount of protein required was calculated by using the equivalent of $15 \mu \mathrm{g}$ protein with a molecular weight of $25 \mathrm{kDa}$ as a standard for obtaining 
equimolar amounts. This amount lies in the linear range of protein amount vs. swelling rate; much higher amounts (> $30 \mathrm{ug}$ ) gave faster rates that were not in the linear range anymore. Control liposomes were prepared by addition of buffer without protein. The next day, $200 \mu \mathrm{l}$ of a $10 \mathrm{mM}$ HEPES-buffer ( $\mathrm{pH}$ 7.0) containing $12 \mathrm{mM}$ stachyose (Sigma) was added to the dried proteoliposomes, followed by careful resuspension. For the uptake assays, 5-10 $\mu \mathrm{l}$ of the proteoliposomes were added to $100 \mu \mathrm{l}$ of a substrate solution in $10 \mathrm{mM}$ HEPES, pH 7.0 and mixed by brief vortexing to generate an exprimental dead time of $\sim 5$ s. Liposome swelling was measured by monitoring changes in optical density at $400 \mathrm{~nm}$ at $5 \mathrm{~s}$ intervals for $60 \mathrm{~s}$. The substrate concentration isosmotic to the intra-liposomal milieu was determined by identifying the concentration of substrate that did not cause a change in OD400 upon dilution in control liposomes. For each curve, the slope $(\Delta \mathrm{OD} / \mathrm{s})$ was calculated for the first $15 \mathrm{~s}$ to obtain the swelling rates. The rate for OmpF in glycine was set to $100 \%$ and all other slopes were correlated to this value. Finally, the used substrate concentrations were as follows: glycine $12 \mathrm{mM}$, glucose $12 \mathrm{mM}$, glutamate $10 \mathrm{mM}$, arginine $8 \mathrm{mM}$, meropenem $8 \mathrm{mM}$, imipenem $10 \mathrm{mM}$, ceftazidime $9 \mathrm{mM}$, cefotaxime $8 \mathrm{mM}$, cefepime $4 \mathrm{mM}$, carbenicillin 6 $\mathrm{mM}$.

Simulation details. The starting structures for the channels were prepared following our usual protocol as described earlier. ${ }^{37}$ We used the improved crystal structure which was solved at a resolution of $1.90 \AA$ (PDB code: 4FRX, URL: https://www.rcsb.org/structure/4FRX). We embedded the system in a POPC (1-palmitoyl-2oleoyl-sn-glycero-3-phosphocholine) lipid bilayer consisting of 238 chains and solvated the system with $\sim 15000$ water molecules. We used the Amber99sb-ildn forcefield parameter for OprD, GAFFlipid model for POPC, and TIP3P model for water. ${ }^{38-40}$ We performed unconstrained MD simulations with the AceMD program. ${ }^{41}$ After proper equilibration, we simulated a periodic box in the NVT ensemble using the Langevin thermostat (300 K), a $9 \AA$ cut-off, and particle mesh Ewald (PME) with fourth order splines, for electrostatic interactions. $^{42}$ We used the Plumed 1.3 plug-in for AceMD for the metadynamics simulations. ${ }^{43}$ We followed the Well-Tempered metadynamics protocol. ${ }^{44}$ To ensure convergence, we constructed the final free energy surface using the 'multiple walkers' method. ${ }^{45}$ The technical details and the protocols have been explained in detail in our previous report. ${ }^{37}$ The free energy surfaces are a result of at least $1.5 \mu$ s of simulation time for the substrates, $2.5 \mu$ s for ceftazidime, and $6 \mu$ s for the neutral spheres.

\section{Electrophysiology: Planar Lipid Bilayer Measurements:}


The measurements were taken with planar lipid membranes. Both chambers (2.5 mL each) of the bilayer apparatus were separated by a $25 \mu \mathrm{m}$ thick Teflon septum. The bilayers were made according to the monolayer technique of Montal and Mueller. ${ }^{6,46}$ An aperture in the septum with a diameter of 70-120 $\mu \mathrm{m}$ was pre-treated with hexadecane dissolved in highly purified n-hexane, at a concentration of $1 \%(\mathrm{v} / \mathrm{v})$. Following prepainting, the chambers were dried for 15-20 min. The standard electrolyte in both compartments contained $10 \mathrm{mM} \mathrm{KCl}$ and $20 \mathrm{mM}$ 2-( $\mathrm{N}$-morpholino)ethanesulfonic acid ( $\mathrm{pH} \mathrm{6.0)}$ for all substrates unless specified. The bilayer was made with 1,2- diphytanoyl-sn-glycerophosphocholine at a concentration of $5 \mathrm{mg} / \mathrm{ml}$ in n-pentane. It should be noted that for technical reasons electrophysiology require lipids in the fluid phase. The purely artificial lipid 1,2- diphytanoyl-snglycerophosphocholine is fully saturated and has no phase transition in a wide range of temperature. Control measurements have been done and in particular porins from bacteria do not show a particular effect of lipid composition. OccK8 was added to both cis and trans sides. Standard Ag/AgCl electrodes were used to detect the ionic current. The cis side electrode of the cell is grounded, whereas the trans side electrode was connected to the headstage of an Axopatch 200B amplifier, used for the Conductance measurements in the voltage clamp mode. Signals were filtered by an on board low pass Bessel filter at $10 \mathrm{kHz}$ and recorded onto a computer hard drive with a sampling frequency of $50 \mathrm{kHz}$. Experiments were repeated at least three times.

\section{Reversal potential measurements}

The conclusions drawn from the conductance experiments were supported by measurements of zero-current membrane potentials $\mathrm{V}_{\text {rev }}$ in the presence of different concentrations of the electrolyte on both sides of the planer lipid bilayer with the OccK8 porin inserted. ${ }^{20}$ After incorporation of OccK8 into the bilayer, single channel currents were recorded at the given membrane voltage $\left(\mathrm{V}_{\text {rev }}\right)$ (control) in buffer containing $10 \mathrm{mM} \mathrm{NaCl}$ and $10 \mathrm{mM}$ MES pH 6.0, followed by cis-side addition of $50 \mathrm{mM}$ antibiotics or the amino acids Glu and Arg.

The current-voltage relationship for the individual experiments was calculated from averaged currents $(n \geq 3)$ at the given voltage (see Figure S10A-D). Standard solutions (control) contained $10 \mathrm{mM} \mathrm{NaCl}$, buffered with $10 \mathrm{mM}$ Mops, $\mathrm{pH}$ 6, cis/trans, (cis is the groundconnected side). After measuring the respective $\mathrm{I}-\mathrm{V}$ curve of the control, the different compounds were added to the cis side at the concentration listed in Table 1 and the respective $\mathrm{I}-\mathrm{V}$ curves were calculated from recordings like those in Figure S10A-D. The relative 
permeability of cations vs anions vs substrate at tri-ionic conditions in Table 1 were obtained by fitting of the experimental I-V-curves with the Goldman-Hodgkin-Katz current equation. ${ }^{20}$, 47,48

\section{Ancillary information.}

Supplementary information accompanies this paper. It includes sequence alignment of OccK8 with other similar proteins, structure of the antibiotics, structural features of OccK8 obtained from simulations, and the details of the electrophysiology experiments.

Data availability. Coordinates and structure factors for OccK8 have been deposited in the Protein Data Bank with accession code 4FRX.

Competing financial interests. The authors declare no competing interests.

Corresponding author Information: Corresponding author: Matteo Ceccarelli. Email: matteo.ceccarelli@dsf.unica.it

Author contributions: S.S., I.B., S.A.G., T.D.A, and M.C. performed MD simulations; M.P. and B.vdB. performed LSA experiments and solved crystal structure; C.S. and D.B. performed proteomics experiments; I.G., R.W. and M.W. performed electrophysiology experiments; M.W., B.vdB. and M.C. designed the work. All authors contributed to writing of the manuscript.

Acknowledgements: Simulations that lead to part of the results in this paper have been performed using the PRACE Research Infrastructure resource Curie based in France at TGCC, under project Tier-0 nr. RA2699. Funding: The research leading to these results was conducted as part of the Translocation consortium (www.translocation.com) and has received support from the Innovative Medicines Initiative Joint Undertaking under Grant Agreement no. 115525, resources which are composed of financial contribution from European Union's seventh framework programme (FP7/2007-2013) and EFPIA companies. S.A.G. and M.P. are funded by EU FP7-PEOPLE-2013-ITN Translocation network Nr. 607694. 


\section{References}

1. Tommasi, R.; Brown, D.; Walkup, G.; Manchester, J.; Miller, A., ESKAPEing the labyrinth of antibacterial discovery. Nature Reviews Drug Discovery 2015, 14 (8), 529-542. DOI: $10.1038 / \mathrm{nrd} 4572$.

2. $\quad$ Payne, D. J.; Gwynn, M. N.; Holmes, D. J.; Pompliano, D. L., Drugs for bad bugs: confronting the challenges of antibacterial discovery. Nature Reviews Drug Discovery 2007, 6 (1), 29-40.

3. Brown, E.; Wright, G., Antibacterial drug discovery in the resistance era. Nature 2016, 529 (7586), 336-343. DOI: 10.1038/nature17042.

4. $\quad$ Payne, D.; Miller, L.; Findlay, D.; Anderson, J.; Marks, L., Time for a change: addressing R\&D and commercialization challenges for antibacterials. Philosophical Transactions of the Royal Society B-Biological Sciences 2015, 370 (1670). DOI: 10.1098/rstb.2014.0086.

5. $\quad$ Eren, E.; Vijayaraghavan, J.; Liu, J.; Cheneke, B. R.; Touw, D. S.; Lepore, B. W.; Indic, M.; Movileanu, L.; Van den Berg, B., Substrate specificity within a family of outer membrane carboxylate channels. PLoS Biology 2012, 10 (1), e1001242.

6. $\quad$ Liu, J.; Eren, E.; Vijayaraghavan, J.; Cheneke, B.; Indic, M.; van den Berg, B.; Movileanu, L., OccK Channels from Pseudomonas aeruginosa Exhibit Diverse SingleChannel Electrical Signatures but Conserved Anion Selectivity. Biochemistry 2012, 51 (11), 2319-2330. DOI: 10.1021/bi300066w.

7. Cheneke, B. R.; van den Berg, B.; Movileanu, L., Analysis of gating transitions among the three major open states of the OpdK channel. Biochemistry 2011, 50 (22), 4987 97. DOI: $10.1021 /$ bi200454j.

8. Z Zahn, M.; Bhamidimarri, S. P.; Baslé, A.; Winterhalter, M.; van den Berg, B., Structural Insights into Outer Membrane Permeability of Acinetobacter baumannii. Structure 2016, 24 (2), 221-31. DOI: 10.1016/j.str.2015.12.009.

9. $\quad$ Scorciapino, M. A.; D'Agostino, T.; Acosta-Gutierrez, S.; Malloci, G.; Bodrenko, I.; Ceccarelli, M., Exploiting the porin pathway for polar compound delivery into Gramnegative bacteria. Future Med Chem 2016. DOI: 10.4155/fmc-2016-0038.

10. Acosta-Gutierrez, S.; Scorciapino, M.; Bodrenko, I.; Ceccarelli, M., Filtering with Electric Field: The Case of E. coli Porins. Journal of Physical Chemistry Letters 2015, 6 (10), 1807-1812. DOI: 10.1021/acs.jpclett.5b00612.

11. Acosta Gutiérrez, S.; Bodrenko, I.; Scorciapino, M. A.; Ceccarelli, M., Macroscopic electric field inside water-filled biological nanopores. Physical Chemistry Chemical Physics 2016, 18 (13), 8855-8864. DOI: 10.1039/c5cp07902k.

12. Ghai, I.; Bajaj, H.; Bafna, J. A.; Damrany Hussein, H. A. E.; Winterhalter, M.; Wagner, R., Ampicillin permeation across OmpF, the major outer membrane channel in E. coli. J Biol Chem 2018. DOI: 10.1074/jbc.RA117.000705.

13. Isabella, Vincent M.; Campbell, Arthur J.; Manchester, J.; Sylvester, M.; Nayar, Asha S.; Ferguson, Keith E.; Tommasi, R.; Miller, Alita A., Toward the Rational Design of Carbapenem Uptake in Pseudomonas aeruginosa. Chemistry \& Biology 2015, 22 (4), 535547. DOI: http://dx.doi.org/10.1016/j.chembiol.2015.03.018.

14. Iyer, R.; Sylvester, M. A.; Velez-Vega, C.; Tommasi, R.; Durand-Reville, T. F.; Miller, A. A., Whole-Cell-Based Assay To Evaluate Structure Permeation Relationships for Carbapenem Passage through the Pseudomonas aeruginosa Porin OprD. ACS Infect Dis 2017, 3 (4), 310-319. DOI: 10.1021/acsinfecdis.6b00197.

15. Dupont, H.; Gaillot, O.; Goetgheluck, A.; Plassart, C.; Emond, J.; Lecuru, M.; Gaillard, N.; Derdouri, S.; Lemaire, B.; de Courtilles, M.; Cattoir, V.; Mammeri, H., Molecular Characterization of Carbapenem-Nonsusceptible Enterobacterial Isolates Collected 
during a Prospective Interregional Survey in France and Susceptibility to the Novel Ceftazidime-Avibactam and Aztreonam-Avibactam Combinations. Antimicrobial Agents and Chemotherapy 2016, 60 (1), 215-221. DOI: 10.1128/AAC.01559-15.

16. Zhanel, G.; Lawson, C.; Adam, H.; Schweizer, F.; Zelenitsky, S.; Lagace-Wiens, P.; Denisuik, A.; Rubinstein, E.; Gin, A.; Hoban, D.; Lynch, J.; Karlowsky, J., CeftazidimeAvibactam: a Novel Cephalosporin/beta-lactamase Inhibitor Combination. Drugs 2013, 73 (2), 159-177. DOI: 10.1007/s40265-013-0013-7.

17. Yamano, Y.; Nishikawa, T.; Komatsu, Y., Outer membrane proteins responsible for the penetration of beta-lactams and quinolones in Pseudomonas aeruginosa. J Antimicrob Chemother 1990, 26 (2), 175-84.

18. Yamano, Y.; Nishikawa, T.; Komatsu, Y., Involvement of the RpoN protein in the transcription of the oprE gene in Pseudomonas aeruginosa. Fems Microbiology Letters 1998, 162 (1), 31-37. DOI: 10.1016/S0378-1097(98)00098-6.

19. Yamano, Y.; Nishikawa, T.; Komatsu, Y., Cloning and Nucleotide-Sequence of Anaerobically Induced Porin Protein-E1 (OPRE) of Pseudomonas aeruginosa PAO1. Molecular Microbiology 1993, 8 (5), 993-1004. DOI: 10.1111/j.1365-2958.1993.tb01643.x. 20. Ghai, I.; Pira, A.; Scorciapino, M.; Bodrenko, I.; Benier, L.; Ceccarelli, M.; Winterhalter, M.; Wagner, R., A General Method to Determine the Flux of Charged Molecules Through Nanopores Applied to ß-Lactamase Inhibitors and OmpF. The journal of physical chemistry letters 2017.

21. Bangham, A. D.; Hill, M. W.; Miller, N. G. A., Preparation and Use of Liposomes as Models of Biological Membranes. In Methods in Membrane Biology, Korn, E. D., Ed. Springer US: Boston, MA: 1974, 1974; pp 1-68.

22. Danelon, C.; Suenaga, A.; Winterhalter, M.; Yamato, I., Molecular origin of the cation selectivity in OmpF porin: single channel conductances vs. free energy calculation. Biophys Chem 2003, 104 (3), 591-603.

23. Hille, B., Ionic Channels of Excitable Membranes. Sinauer Ass. Inc.: Sunderland, Ma 01375, 2001; Vol. 3.

24. Meuser, D.; Splitt, H.; Wagner, R.; Schrempf, H., Exploring the open pore of the potassium channel from Streptomyces lividans. FEBS Letters 1999, 462 (3), 447-52.

25. Hodgkin, A. L.; Katz, B., The effect of sodium ions on the electrical activity of the giant axon of the squid. $J$ Physiol 1949, 108 (1), 37-77.

26. Goldman, D. E., Potential, Impedance, and Rectification in Membranes. Journal of General Physiology 1943, 21 (1), 37-60. DOI: 10.1085/jgp.27.1.37.

27. Jaouen, T.; Coquet, L.; Marvin-Guy, L.; Orange, N.; Chevalier, S.; De, E., Functional characterization of Pseudomonas fluorescens OprE and OprQ membrane proteins.

Biochemical and Biophysical Research Communications 2006, 346 (3), 1048-1052. DOI: 10.1016/j.bbrc.2006.06.013.

28. Ferrara, L.; Wallat, G.; Moynie, L.; Dhanasekar, N.; Aliouane, S.; Acosta-Gutierrez, S.; Pages, J.; Bolla, J.; Winterhalter, M.; Ceccarelli, M.; Naismith, J., MOMP from Campylobacter jejuni Is a Trimer of 18-Stranded beta-Barrel Monomers with a Ca2+ Ion Bound at the Constriction Zone. Journal of Molecular Biology 2016, 428 (22), 4528-4543. DOI: 10.1016/j.jmb.2016.09.021.

29. Benkerrou, D.; Ceccarelli, M., Free energy calculations and molecular properties of substrate translocation through OccAB porins. Phys Chem Chem Phys 2018. DOI: 10.1039/c7cp08299a.

30. Scorciapino, M. A.; Acosta-Gutierrez, S.; Benkerrou, D.; D'Agostino, T.; Malloci, G.; Samanta, S.; Bodrenko, I.; Ceccarelli, M., Rationalizing the permeation of polar antibiotics into Gram-negative bacteria. J Phys Condens Matter 2017, 29 (11), 113001. DOI: 10.1088/1361-648X/aa543b. 
31. Bajaj, H.; Acosta Gutierrez, S.; Bodrenko, I.; Malloci, G.; Scorciapino, M. A.; Winterhalter, M.; Ceccarelli, M., Bacterial Outer Membrane Porins as Electrostatic Nanosieves: Exploring Transport Rules of Small Polar Molecules. ACS Nano 2017, 11 (6), 5465-5473. DOI: 10.1021/acsnano.6b08613.

32. Emsley, P.; Cowtan, K., Coot: model-building tools for molecular graphics. Acta Crystallogr D Biol Crystallogr 2004, 60 (Pt 12 Pt 1), 2126-32. DOI:

10.1107/S0907444904019158.

33. Malloci, G.; Vargiu, A. V.; Serra, G.; Bosin, A.; Ruggerone, P.; Ceccarelli, M., A Database of Force-Field Parameters, Dynamics, and Properties of Antimicrobial Compounds. Molecules 2015, 20 (8), 13997-4021. DOI: 10.3390/molecules200813997.

34. D'Agostino, T.; Salis, S.; Ceccarelli, M., A kinetic model for molecular diffusion through pores. Biochim Biophys Acta 2016, 1858 (7 Pt B), 1772-7. DOI: 10.1016/j.bbamem.2016.01.004.

35. McCoy, A. J.; Grosse-Kunstleve, R. W.; Adams, P. D.; Winn, M. D.; Storoni, L. C.; Read, R. J., Phaser crystallographic software. J Appl Crystallogr 2007, 40 (Pt 4), 658-674. DOI: $10.1107 /$ S0021889807021206.

36. Zwart, P. H.; Afonine, P. V.; Grosse-Kunstleve, R. W.; Hung, L. W.; Ioerger, T. R.; McCoy, A. J.; McKee, E.; Moriarty, N. W.; Read, R. J.; Sacchettini, J. C.; Sauter, N. K.; Storoni, L. C.; Terwilliger, T. C.; Adams, P. D., Automated structure solution with the PHENIX suite. Methods Mol Biol 2008, 426, 419-35. DOI: 10.1007/978-1-60327-058-8_28.

37. Samanta, S.; Scorciapino, M. A.; Ceccarelli, M., Molecular basis of substrate translocation through the outer membrane channel OprD of Pseudomonas aeruginosa. Physical Chemistry Chemical Physics 2015, 17 (37), 23867-23876. DOI: 10.1039/c5cp02844b.

38. Lindorff-Larsen, K.; Piana, S.; Palmo, K.; Maragakis, P.; Klepeis, J. L.; Dror, R. O.; Shaw, D. E., Improved side-chain torsion potentials for the Amber ff99SB protein force field. Proteins: Structure, Function, and Bioinformatics 2010, 78 (8), 1950-1958. DOI: 10.1002/prot.22711.

39. Dickson, C. J.; Rosso, L.; Betz, R. M.; Walker, R. C.; Gould, I. R., GAFFlipid: a General Amber Force Field for the accurate molecular dynamics simulation of phospholipid. Soft Matter 2012, 8 (37), 9617-9627. DOI: 10.1039/c2sm26007g.

40. Jorgensen, W. L.; Chandrasekhar, J.; Madura, J. D.; Impey, R. W.; Klein, M. L., Comparison of simple potential functions for simulating liquid water. Journal of Chemical Physics 1983, 79 (2), 926-935. DOI: doi:http://dx.doi.org/10.1063/1.445869.

41. Harvey, M. J.; Giupponi, G.; Fabritiis, G. D., ACEMD: Accelerating Biomolecular Dynamics in the Microsecond Time Scale. Journal of Chemical Theory and Computation 2009, 5 (6), 1632-1639. DOI: 10.1021/ct9000685.

42. Darden, T.; York, D.; Pedersen, L., Particle Mesh Ewald - an N.Log(N) Method for Ewald Sums in Large Systems. Journal of Chemical Physics 1993, 98 (12), 10089-10092. 43. Bonomi, M.; Branduardi, D.; Bussi, G.; Camilloni, C.; Provasi, D.; Raiteri, P.; Donadio, D.; Marinelli, F.; Pietrucci, F.; Broglia, R. A., PLUMED: A Portable Plugin for Free-Energy Calculations with Molecular Dynamics. Computer Physics Communications 2009, 180 (10), 1961-1972.

44. Barducci, A.; Bussi, G.; Parrinello, M., Well-Tempered Metadynamics: A Smoothly Converging and Tunable Free-Energy Method. Physical Review Letters 2008, 100 (2), 020603.

45. Raiteri, P.; Laio, A.; Gervasio, F. L.; Micheletti, C.; Parrinello, M., Efficient Reconstruction of Complex Free Energy Landscapes by Multiple Walkers Metadynamics. Journal of Physical Chemistry B 2006, 110 (8), 3533-3539. 
46. Bajaj, H.; Tran, Q.; Mahendran, K.; Nasrallah, C.; Colletier, J.; Davin-Regli, A.; Bolla, J.; Pages, J.; Winterhalter, M., Antibiotic Uptake through Membrane Channels: Role of Providencia stuartii OmpPst1 Porin in Carbapenem Resistance. Biochemistry 2012, 51 (51), 10244-10249. DOI: 10.1021/bi301398j.

47. Citak, F.; Ghai, I.; Rosenkötter, F.; Benier, L.; Winterhalter, M.; Wagner, R., Probing transport of fosfomycin through substrate specific OprO and OprP from Pseudomonas aeruginosa. Biochemical and biophysical research communications 2017.

48. $\quad$ Ghai, I.; Winterhalter, M.; Wagner, R., Probing transport of charged $\beta$-lactamase inhibitors through OmpC, a membrane channel from E. coli. Biochemical and Biophysical Research Communications 2017, 484 (1), 51-55.

\section{Tables}

Table 1. Measured reversal potentials and tri-ionic permeability ratios for different substrates through the OccK8 porin of Pseudomonas aeruginosa. Calculated conductance and turnover rates (n/s) of OccK8 for different substrates are shown in two columns in the right.

\begin{tabular}{|c|c|c|c|c|c|c|c|}
\hline Substrate* & $\begin{array}{c}C_{\mathrm{NaCl}} \\
(m M) \\
\text { (cis/trans) }\end{array}$ & $\begin{array}{c}C_{i} \\
(m M) \\
\text { (cis) }\end{array}$ & $V_{r e v}^{\exp }(m V)$ & $\begin{array}{c}\text { charg } \\
\text { el } \\
\text { pH6* }\end{array}$ & $\begin{array}{l}P_{\mathrm{Na}^{+i} / P_{i} i \dot{ }} \\
\text { (calc)** }\end{array}$ & $\begin{array}{c}\text { conductance } \\
G(f S) * * * \\
10 \mathrm{mM}\end{array}$ & $\begin{array}{c}\text { rate } \\
(\mathrm{mol} / \mathrm{s})^{\star \star \star *} \\
(\text { at } 10 \mu \mathrm{M} \\
\left.\mathrm{V}_{\mathrm{m}}=10 \mathrm{mV}\right)\end{array}$ \\
\hline $\mathrm{NaCl}$ & $600 / 100$ & & $8 \pm 2$ & & $1.55: 1$ & - & - \\
\hline $\begin{array}{c}(\text { Ceftazidime })^{-6 i} \\
\mathrm{Na}^{+i i}\end{array}$ & $10 / 10$ & 50 & $25.7 \pm 3.5$ & -1 & 1.55:1:0.27 & 64 & 0.4 \\
\hline $\begin{array}{c}(\text { Cefotaxime })^{-i i} \\
\mathrm{Na}^{+i i}\end{array}$ & $10 / 10$ & 50 & $27 \pm 3$ & -1 & $1.55: 1: 0.20$ & 48 & 0.1 \\
\hline $\begin{array}{c}(G l u)^{-i 6} \\
N a^{+i 6}\end{array}$ & $10 / 10$ & 50 & $22 \pm 2.5$ & -1 & $1.55: 1: 0.38$ & 92 & 0.6 \\
\hline $\begin{array}{c}(\text { Carbenicilin })^{2-i} \\
i \dot{i} i\end{array}$ & $10 / 10$ & 50 & $36 \pm 5$ & -1 & 1.55:1:0.01 & 5.9 & 0.04 \\
\hline $\operatorname{Arg}^{+i C l^{-i c} i}$ & $10 / 10$ & 50 & $-26 \pm 3$ & +1 & 1.55:1:0.06 & 23 & 0.1 \\
\hline
\end{tabular}

* http://www.dsf.unica.it/translocation/db/

** The permeability ratio of $P_{\mathrm{Na}^{+i} / P_{i} i \text { i }}$ for Occk8 which has been determined independently under bionic conditions and was fixed during fitting of $\mathrm{V}_{\text {rev }}$ (tri-ionic).

Fitting of the obtained reversal potentials using the GHK-current equation (see SI for details). *** The conductance values were obtained from the linear part of the slope of the calculated $\mathrm{I}-\mathrm{V}$ curves for the individual substrate at $10 \mathrm{mM}$ concentrations $(\mathrm{pH} 6)$ each. (for details see $\mathrm{SI})$.

**** The turnover number, or number of molecules/second, for the different substrates was calculated from the conductance of the OccK8 channel for the substrates at $10 \mu \mathrm{M}$ concentration as described in detail in the SI. 


\begin{tabular}{|c|c|c|}
\hline & & OccK8 \\
\hline \multicolumn{3}{|l|}{ Data Collection } \\
\hline Beamline & & NSLS X25 \\
\hline Wavelength & & 1.10 \\
\hline Space Group & & $\mathrm{C} 2$ \\
\hline \multicolumn{3}{|l|}{ Cell Dimensions } \\
\hline & a,b,c $(\AA)$ & $138.4,90.1,103.6$ \\
\hline & $\alpha, \beta, \gamma\left(\left(^{\circ}\right)\right.$ & $90,92.5,90$ \\
\hline Resolution $(\AA)$ & & $50-1.9$ \\
\hline Completeness $^{1}$ & & $99.3(98.6)$ \\
\hline Redundancy & & $3.5(3.4)$ \\
\hline$I / \sigma$ & & $9.2(1.9)$ \\
\hline $\mathrm{R}_{\mathrm{sym}}$ & & $11.8(55.3)$ \\
\hline \multicolumn{3}{|l|}{ Refinement } \\
\hline Resolution $(\AA)$ & & $15-1.9$ \\
\hline Unique Reflections ${ }^{2}$ & & 99141 (1994) \\
\hline $\mathrm{R}_{\text {work }} / \mathrm{R}_{\text {free }}{ }^{3}$ & & $21.3 / 24.7$ \\
\hline \multicolumn{3}{|l|}{ R.m.s.d. } \\
\hline & bond lengths $(\AA)$ & 0.007 \\
\hline & bond angles $\left({ }^{\circ}\right)$ & 1.14 \\
\hline \multicolumn{3}{|l|}{ Average B-factors $\left(\AA^{2}\right)$} \\
\hline & $\begin{array}{c}\text { protein/detergent/ } \\
\text { water }\end{array}$ & 18.2/39.2/29.7 \\
\hline \multicolumn{3}{|l|}{ Ramachandran } \\
\hline & $\begin{array}{c}\text { favored/allowed/ } \\
\text { outliers (\%) }\end{array}$ & $96.2 / 3.5 / 0.3$ \\
\hline Molprobity Clashscore & & 6.6 \\
\hline
\end{tabular}

Table 2. Data collection and refinement statistics of OccK8

${ }^{1}$ Values in parentheses are for the highest resolution shell

${ }^{2}$ Value in parentheses is the number of reflections used to calculate $\mathrm{R}_{\text {free }}$ ${ }^{3} \mathrm{R}_{\text {work }}=\sum|\mathrm{Fo}-\mathrm{Fc}| / \sum$ Fo. $\mathrm{R}_{\text {free }}$ is the cross-validation of the R-factor 
For Table of Contents Use Only

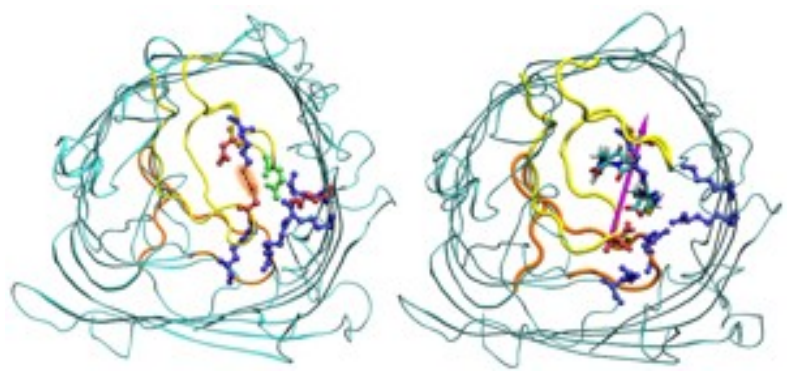

